\title{
Novel Bacteria Foraging Optimization for Energy-efficient Communication in Wireless Sensor Network
}

\author{
Hemavathi $\mathbf{P}^{1}$, Nandakumar A $\mathbf{N}^{2}$ \\ ${ }^{1}$ Jain University, India \\ ${ }^{2}$ Department of Computer Science and Engineering, New Horizon College of Engineering, India
}

\begin{tabular}{l} 
Article Info \\
\hline Article history: \\
Received Dec 28, 2017 \\
Revised Jul 17, 2018 \\
Accepted Jul 29, 2018 \\
\hline
\end{tabular}

\section{Keyword:}

Bacterial foraging Energy efficiency Optimization Swarm intelligence Wireless sensor network

\begin{abstract}
Optimization techniques based on Swarm-intelligence has been reported to have significant benefits towards addressing communication issues in Wireless Sensor Network (WSN). We reviewed the most dominant swarm intelligence technique called as Bacteria Foraging Optimization (BFO) to find that there are very less significant model towards addressing the problems in WSN. Therefore, the proposed paper introduced a novel BFO algorithm which maintains a very good balance between the computational and communication demands of a sensor node unlike the conventional BFO algorithms. The significant contribution of the proposed study is to minimize the iterative steps and inclusion of minimization of both receiving / transmittance power in entire data aggregation process. The study outcome when compared with standard energy-efficient algorithm was found to offer superior network lifetime in terms of higher residual energy as well as data transmission performance.
\end{abstract}

Copyright () 2018 Institute of Advanced Engineering and Science. All rights reserved.

\section{Corresponding Author:}

Hemavathi $\mathrm{P}$

Department of Computer Science and Engineering,

Bangalore Institute of Technology, Bangalore, India

Email: hemavathi.research@gmail.com

\section{INTRODUCTION}

Wireless Sensor Network (WSN) has been a dominant topic of research in wireless network from more than a decade owing to its ongoing research challenges and its endless opportunities [1]. There are various research problems associated with WSN e.g. security issues [2], routing issues [3], energy issues [4], traffic-related issues [5], design issues [6], etc. In parallel to the problems, there is also various researchbased solution that has been consistently evolving to solve this. Owing to design constraints of a sensor node, an optimization-based solution is the most preferred approach in addressing issues in WSN. Out of various forms of research techniques in optimization [7], swarm-intelligence based optimization is now becoming a trend owing to following benefits: inclusion of cognitive and social intelligence that offers better granularity in solving the optimization problems, better convergence performance compared to other non-bio inspired techniques and simpler interpretation of outcomes owing to its match with certain living organism [8].

Among all this, the proposed research work has emphasized on using Bacteria Foraging Optimization (BFO), which is based on foraging behaviour of a bacteria [9]. The fundamental strategy of the $\mathrm{BFO}$ algorithm is to permit the cell i.e. bacteria for aggregating stochastically the swarm and leading it to the position of optima as means to process information within a sensor node. In order to do this, there are sequences of operations being performed by the BFO. The first operation is known as chemotaxis which the system de-rates the cellular cost owing to the nearness to other bacteria while the movement of the cells is carried out towards the processed surface cost one by one. This operation is highly iterative in order to accomplish better outcome. The second operation is called as reproduction which means the continuation of a particular bacteria to next generation if they were witnessed to do well over a sample period of time. 
The third operation is called as elimination-dispersal where the system rejects the cells and considers accepting novel samples of random origin with an inclusion of very minimal probability. A typical $\mathrm{BFO}$ algorithm is originally meant for solving optimization problem with continuous function. Owing to its recursive operation, it always works on a given loops in order to construct various form of explicit search operation [10]. The design process also uses different forms of coefficients for modeling swarming environment e.g. depth and width of attracting signal, width of repellant signal, and height of repellent signal. The conventional concept considers that height of repellant signal is always equivalent to depth of attractive signal. Adoption of a smaller step size in designing BFO is very common for continuing the search for an elite outcome. Conventional BFO algorithm also considers rejecting the half of cell population excusively during the reproduction which are reported to have lower health signals.

The concept of BFO was already used in solving various optimization-based problems. However, inspite of such potential concept of optimization, its utilization towards WSN is yet to be witnessed as there is no standard research work or any benchmarked model being ever reported to be using BFO in WSN. Some of the research attempts of BFO towards WSN are mainly to perform selection of $\mathrm{CH}$ with no particular focus on its iterative operation. Therefore, the proposed research work introduces a technique which enhances the conventional BFO algorithm in order to optimize both computational and communication performance of a cellular node. The prime idea of the proposed paper is also to minimize the amount of energy depletion by making use of its iterative operation involved in enhanced BFO design principle. Section 1.1 discusses about the existing literatures where different techniques of BFO in different optimization issues are discussed followed by discussion of research problems in Section 1.2 and proposed solution in 1.3. Section 2 discusses about proposed optimization algorithm implementation followed by discussion of result analysis in Section 3. Finally, the conclusive remarks are provided in Section 4.

\subsection{Background}

This section discusses the existing research approaches used for implementing the typical swarm intelligence of Bacteria Foraging Optimization (BFO). Gupta et al. [11] have presented a study where BFO was used for minimizing the degree of color in order to enhance the quantization process of a colored image. Dasgupta et al. [12] have enhanced the conventional BFO in order to solve the optimimization problems associated with high-dimensional data. Chen et al. [13] have addressed the convergence issue of conventional BFO by incorporating the automatic fine tuning of run length during execution of an algorithm. Wei et al. [14] have presented an adaptive BFO algorithm along with conventional search optimization technique in order to enhance the accuracy of search. Similar direction of adaptiveness has been also implemented by Nasir et al. [15] considering different forms of modalities in benchmarking process. Panda and Naik [16] have implemented a cross-over mechanism of genetic algorithm for improving the operations in BFO technique. Mangaraj et al. [17] have used BFO for enhancing the design process involved in antenna. Mao et al. [18] have combinely used particle swarm optimization along with BFO in order to improve the optimization performance involved in design of numerical function. Monajemi et al. [19] have applied diffusion adaption for modeling the motivity concept of bacteria in order to investigate the networks of bacteria.

Similar approach of adaptation along with enhancement in reproduction step is carried out by Daas et al. [20]. BFO algorithm was also reported to be used for optimizing the traffic performance under the congestion state as seen in work of Jain et al. [21]. Usage of BFO was reported to be used for retaining uniform output of power in grid system as seen in the work of Mishra et al. [22]. Munoz et al. [23] have presented a brief discussion of using the complex functions involved in BFO using statistical analysis. Okaeme and Zanchetta [24] have used BFO for optimizing the performance of the controller design involved in electrical drives. BFO was also reported to be used for electric vehicles for enhancing the energy efficiency as seen in the work of Samanta et al. [25]. BFO algorithms have also been investigated in sensor network apart from other optimization problems.Ari et al.

C. Samanta et. al. Have used BFO algorithm for assisting in mobile sensing operation in Wireless Sensor Network (WSN) [26]. Lalwani and Das have used BFO for performing selection of the cluster head mainly targeting to improve the routing performance [27]. The technique presented y Li et al have nearly used the similar mechanism of BFO along with quantum computing for enhancibf its stability rate during convergence of the outcome [28]. Pitchaimanickam and Radhakrishnan have have applied BFO technique on clusterhead selection process using simulation-based approach where the study outcome shows enhanced protocol operation. Usage of BFO was also reported in the work of Zhao et al. [30] in the area of robotics using nanorobots. Therefore, this background information eventually proved that BFO was investigated for multiple case studies of optimization problems. The next section outlines the issues associated with existing research work. 


\subsection{The Problem}

The significant research problems are as follows:

a. A typical BFO algorithm suffers from convergence problems as well as it involves too much iterative steps in its sub-operations that has not been found to be addressed.

b. Studies towards applying BFO algorithm towards optimizing the performance of WSN is less explored as compared to other optimization problems.

c. There are few research implementations towards balancing the computation and communication performance in WSN using BFO algorithm.

d. There is no single research work that confirmly minimize the dissipated power of sensor nodes during data aggregation process using BFO.

Therefore, the problem statement of the proposed study can be stated as "Maintaining a uniform balance between the computational performance of BFO algorithm and optimal energy-efficient data delivery performance in WSN is challenging to design and implement."

\subsection{The Proposed Solution}

Extending out prior work [31], the proposed system aims to perform optimization of the energy performance of the sensor node in WSN by minimizing the iterative steps in order to offer faster convergence performance in Bacteria Foraging Optimization. Figure 1 highlights the system model of the proposed optimization.

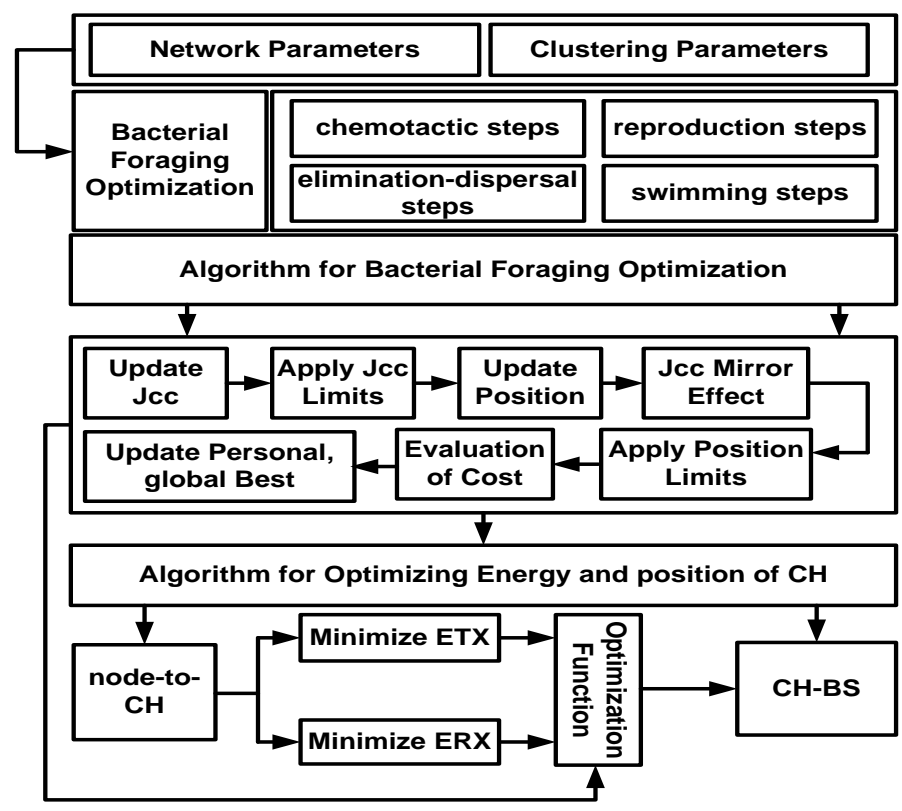

Figure 1. Proposed System Model

Figure 1 highlights that system takes input of network and clustering parameters of the WSN and constructs a novel bacterial foraging optimization technique in order to consecutively construct a mechanism that can optimally select a clusterhead in order to ensure that it performs minimization of the energy dissipation with respect to both transmittance and receiving energy. The proposed system not only minimizes the iterative steps of optimization but also reduces the transmittance energy twice in each steps that results in significant improvement of network lifetime as well as it retains a good balance between energy and throughput. The next section illustrates about algorithm implementation.

\section{ALGORITHM IMPLEMENTATION}

This part of the paper outlines the optimization algorithm for bacterial foraging. The proposed system basically introduced a core optimization algorithm that is used for two motives i.e. i) performing optimization and ii) minimization of energy factor involved, and iii) selection of an effective cluster heads. This section illustrates the core algorithm by splitting it in two sub-modules. 


\subsection{Algorithm for Bacterial Foraging Optimization}

It has been already found that conventional usage of bacterial foraging behaviour calls for much slower convergence rate inspite of its elite outcomes. Therefore, the essential focus of the proposed system is to ensure that convergence rate is speedened up for faster decision making during optimization process. The algorithm takes the input of $N$ (Number of Nodes), $p$ (probability of selecting $\mathrm{CH}$ ), $\mathrm{E}_{\mathrm{o}}$ (Initialized Energy), $\mathrm{N}_{\mathrm{c}}$ (Number of Chemotactic Steps), $\mathrm{N}_{\mathrm{re}}$ (Number of reproductive steps), $\mathrm{N}_{\mathrm{ed}}$ (Number of elimination-dispersal steps), $\mathrm{N}_{\mathrm{s}}$ (Number of swimming steps), $S$ (Number of Bacteria) which after processing yields an outcome of cost $_{\text {best }}$ (best cost of routing). The significant steps of the algorithms are as follows:

\section{Algorithm for Bacterial Foraging Optimization}

Input: $\mathrm{N}, \mathrm{p}, \mathrm{E}_{\mathrm{o}}, \mathrm{N}_{\mathrm{c}}, \mathrm{N}_{\mathrm{re}}, \mathrm{N}_{\mathrm{ed}}, \mathrm{N}_{\mathrm{s}}, \mathrm{S}$

Output: cost best

Start

1. init $N, p, E_{o}, N_{c}, N_{r e}, N_{e d}, N_{s}, S$

2. For $i=1: S$

3. Compute bac(pos, $\mathbf{J}_{\mathrm{cc},}$ cost)

4. If $\operatorname{bac}\left(\right.$ cost $\left._{\text {best }}\right)<$ cost $_{\text {gbest }}$

5. $\quad$ gbest $\rightarrow$ bac(best)

6. End

7. cost $_{\text {best }} \rightarrow\left[\mathrm{N}_{\mathrm{ed}} * \mathrm{~N}_{\mathrm{re}} * \mathrm{~N}_{\mathrm{c}}\right]$

8. For $l=1: \mathrm{N}_{\mathrm{ed}}$

9. For $\mathrm{k}=1: \mathrm{N}_{\mathrm{re}}$

10. $\quad$ For $\mathrm{j}=1: \mathrm{Nc}$

11. For $\mathrm{i}=1: \mathrm{S}$

12. update $\mathrm{J}_{\mathrm{cc}}$

13. Apply $\mathrm{J}_{\mathrm{cc}}$ limits

14. Update position

$15 . \quad \mathrm{J}_{\mathrm{cc}}$ Effect

16. Apply Position Limits

17. If $\mathrm{bac}\left(\right.$ cost $\left._{\text {best }}\right)<$ cost $_{\text {best }}$

18. $\quad$ gbest $\rightarrow$ bac(Best)

$19 . \quad$ End

20. End

21. End

22. End

23. cost $_{\text {best }} \rightarrow$ gbest

End

The algorithm computes number of cluster head Nch to be product of probability $\mathrm{p}$ and number of nodes $\mathrm{N}$. The variable attribute of bacterial foraging e.g. size $\left(\mathrm{V}_{\text {size }}\right)$, minimum $\left(\mathrm{V}_{\min }\right)$ and maximum $\left(\mathrm{V}_{\max }\right)$ values are initialized. The lower bound $V_{\min }$ and upper bound $V_{\max }$ is initialized to be 0 and 100 respectively. A structure of bacteria $b a c$ is considered by position pos, cost, objective function $\mathrm{J}_{\mathrm{cc}}$, best position and cost ( pos $_{\text {best }}$, cost $\left._{\text {best }}\right)$. For all number of bacteria (S) in Line-2, the cost of bacteria is computed with respect to its nutrient function considering its respective position. This makes the process less iterative compared to conventional approach and makes the convergence faster. If the best cost of the bacteria is found less than cost of the global best than best value of bacteria is allocated as global best (Line-5). The computation of best cost is carried out by product of i) Number of elimination-dispersal steps, ii) Number of reproductive steps, and iii) Number of Chemotactic Steps (Line-7).

The algorithm than apply a very unique steps of non-recursive elimination dispersal step (Line-8), reproductive step (Line-9), and chemotactic step (Line-10). The updating of the objective function $\mathrm{J}_{\mathrm{cc}}$ (Line-12), applying the limits of $\mathrm{J}_{\mathrm{cc}}$ (Line-13), updating positional information (Line-14), and applying positional limits (Line-15). If the best cost of the bacteria is found less than the cost of the global best gbest than the best solution of the bacteria is considered as the global best (Line-18). This process of optimization is applied for efficient selection of clusterhead where a nearest node is explored for the all the cluster node. For all the number of clusterhead, the distance from all the cluster node to its respective member node is computed followed by removal of priorly selected cluster head and searcgh for nearest node to consecutive cluster heads.

\subsection{Algorithm for Optimizing Energy and position of $\mathbf{C H}$}

This algorithm is mainly responsible for optimizing the energy factor of the sensor node and to perform selection of clusterhead considering its positional information. The algorithm takes the input of node 
position ( $\mathrm{x}_{\text {node }}, \mathrm{y}_{\text {node }}$ ) that after processing generates an outcome of $\mathrm{E}_{\mathrm{OTX}}$ (Optimized Transmitted Energy), $\mathrm{E}_{\mathrm{ORX}}\left(\right.$ Optimized Received Energy), pos( (sol $\left._{\text {best }}\right)$ (best position of $\left.\mathrm{CH}\right)$. The steps involved in this algorithm are as follows:

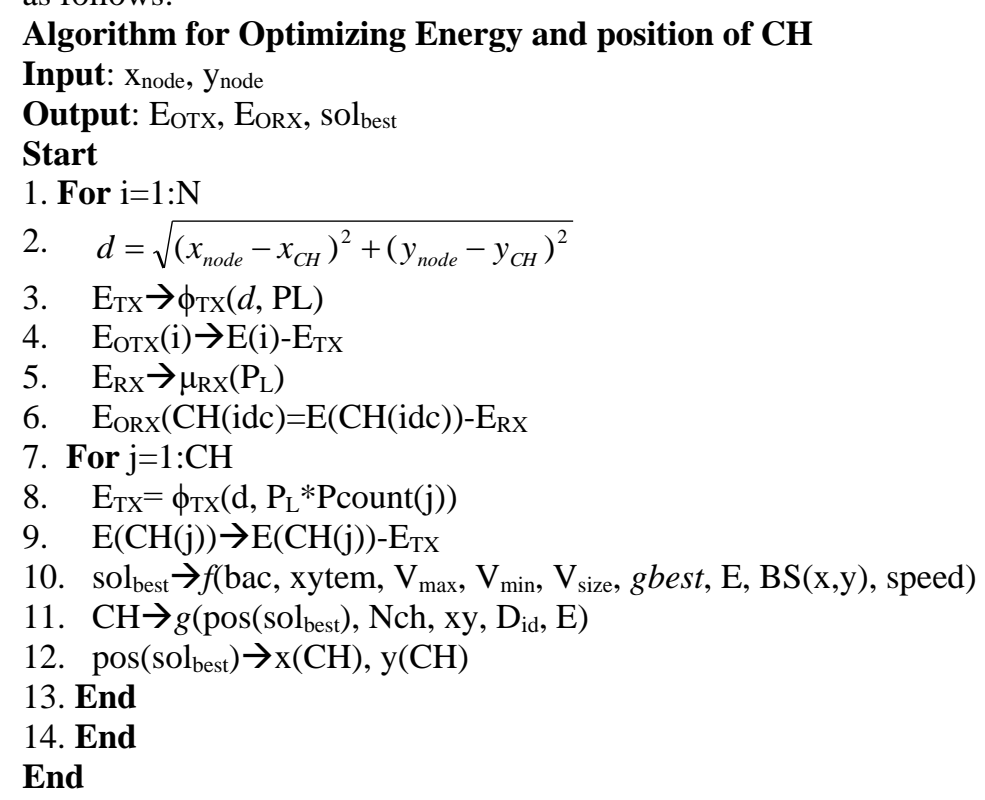

The algorithm mainly emphasizes on two different operations i.e. performing node-to-clusterhead communication (Line-1 to Line-6) and clusterhead-to-base station communication (Line-7 to Line12) in order to apply optimization process during complete stages of data aggregation. For all the nodes (Line-1), Euclidean distance $d$ is computed between the node position $\left(\mathrm{x}_{\text {node }}, \mathrm{y}_{\text {node }}\right)$ and clusterhead position $\left(\mathrm{x}_{\mathrm{CH}}, \mathrm{y}_{\mathrm{CH}}\right)$ (Line-2). The study uses standard first order radio-energy model $\phi_{\mathrm{TX}}$ in order to compute the transmittance energy $\mathrm{E}_{\mathrm{TX}}$ (Line-3), where the transmittance energy is lowered on each iteration round (Line-4) in order to

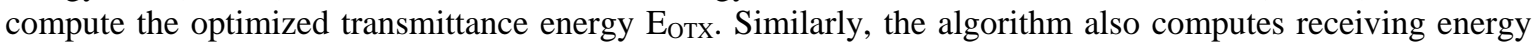
of the clusterhead (Line-5) followed by computation of optimized receiving energy E ERX (Line-6). It can be also seen that computation of both optimized transmittance and receiving energy is carried out considering original data packet $\mathrm{P}_{\mathrm{L}}$. The next phase of the implementation of the algorithm considers only the cluster heads (Line-7 onwards).

The computation of transmittance energy $\mathrm{E}_{\mathrm{TX}}$ is carried out for all the clusterheads only considering distance between the clusterhead and base station $d$, data packet $\mathrm{P}_{\mathrm{L}}$, and number of clusterheads $\mathrm{P}_{\text {count }}$ (Line-8). Even in this step, the algorithm performs minimization of the transmittance energy $\mathrm{E}_{\mathrm{TX}}$. Therefore, the proposed system performs dual steps minimization of transmittance energy that significantly maximizes the network lifetime. Further, the algorithm applies a clustering function $f$ on the basis of bacterial foraging with respect to bacteria bac, xytemp a variable to eliminate died node considering position of all nodes which is about to complete deplete its energy, $\mathrm{V}_{\max }, \mathrm{V}_{\min }$, gbest, energy, position of base station, and speed (Line-10).

This is considered as best solution. Another optimal function $g$ is constructed in Line-11 that considers the best position as per foraging algorithm, number of cluster head, identity of died node $\mathrm{D}_{\text {id, }}$, and energy E. Therefore, Line-11 is considered as the first logical condition for the selection of clusterhead. The second logical condition for the selection of clusterhead is carried out on the basis of position with respect to best solution sol $_{\text {best. }}$. A closer look into this algorithm shows that proposed system mainly contributes towards i) minimizes the iterative steps of bacterial foraging process, ii) offer multiple conditions for selecting clusterhead, iii) optimize the mechanism of first order radio-energy modeling for reliable energy computation to further claim the applicability in real-time sensors.

\section{RESULT ANALYSIS}

As the proposed system focuses on optimization in WSN therefore the assessment is carried out with respect to network lifetime as well as communication performance. For this purpose, we consider residual energy as well as throughput in order to assess the energy performance and data aggregation performance in WSN. The study outcome is assessed for 100 sensor nodes with $0.05 \%$ probability of selection of clusterhead, 0.5 Joule of initialized energy, and 2000 bits of data packet size. The attribites of the bacterial foraging are 100 chemotactic steps, 4 reproduction steps, 2 steps of elimination-dispersal, and 4 
steps of swimming over 100 bacteria. The study also considers width of attractant signal as 0.99 , depth of attractant signal that is generated by bacteria as 1.5, and hight of repellant signal between two bacteria as 2.0.

The study outcome considers comparing its outcome with the standard LEACH algorithm for benchmarking From the study outcome as shown in Figure 2 and Figure 3, it shows that proposed optimization techniques offers exponentially better outcome as compared to existing LEACH. Figure 2 shows sustainance of nodes in LEACH is only till 700 rounds with steep declination of power whereas the proposed system offers quite a predictive pattern of linear pattern of energy dissipation. Figure 3 shows that increased in throughput is limited till 300 iterations and than all the nodes dies by 700 rounds; however, proposed system offers significant linear behaviour of throughput enhancement. Hence, proposed system is highly recommended for any form of emergency-based applications or any other applications where power demands are too high in WSN. Hence, proposed system proved to offer better algorithm performance with respect to energy optimization.

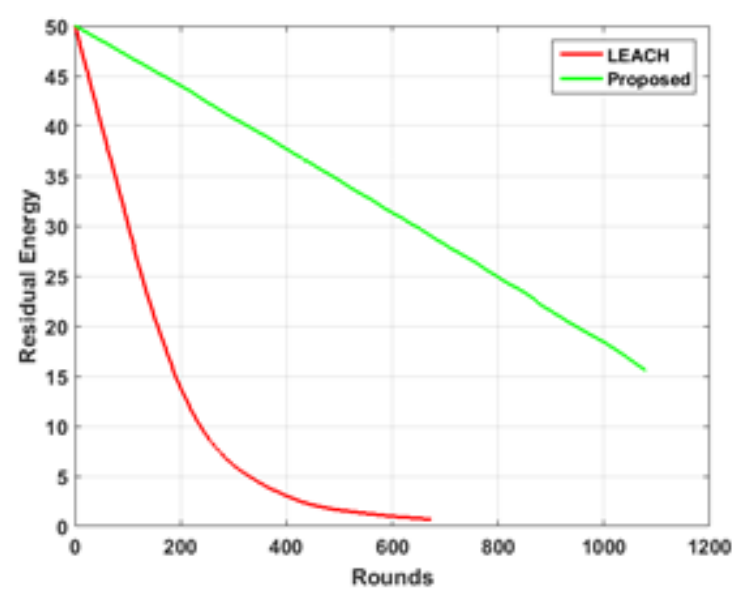

Figure 2. Comparative Analysis of Residual Energy

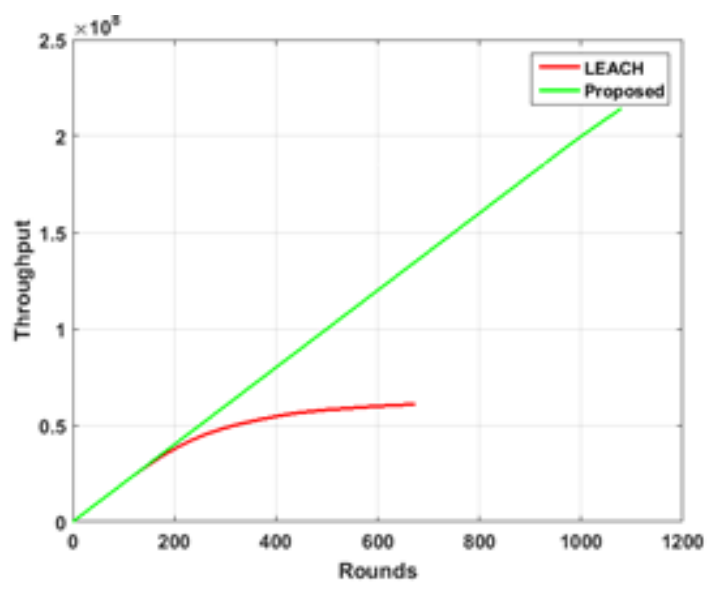

Figure 3. Comparative Analysis of Throughput

\section{CONCLUSION}

This paper has presented a very simple and yet highy effective optimization algorithm that has proved that it is feasible to perform enhancement to bacterial foraging optimization techniques. After reviewing the existing techniques of bacterial foraging techniques, it has been found that existing iterative steps of such algorithm offers good optimization but at the cost of computational complexity owing to inclusion of iterations. The proposed system contributes to an algorithm to have faster convergence accomplishing only within 0.24462 seconds in core i3 processor where as for LEACH it is found to be 0.87666 seconds. Although, we have checked for higher iteration rounds for assessing its outcome, our algorithm is quite predictive in its nature and doesn't require even to be assessed for $10 \%$ of total iterations. Hence, applicability of the algorithm is quite good for energy-demanded application in WSN as it exhibits good balance between energy conservation and increased throughput performance.

\section{REFERENCES}

[1] Rezazadeh, Javad. "Fundamental metrics for wireless sensor networks localization." International Journal of Electrical and Computer Engineering 2.4 (2012): 452.

[2] Anwar, Raja Waseem, et al. "Security in Wireless sensor network: Approaches and Issues." Indonesian Journal of Electrical Engineering and Computer Science 15.3 (2015): 584-590.

[3] Khan, Abdul Waheed, Abdul Hanan Abdullah, and Javed Iqbal Bangash. "Issues towards Efficient Time Synchronization in Wireless Sensor Networks." Indonesian Journal of Electrical Engineering and Computer Science 12.10 (2014): 7509-7522.

[4] N. Abbi and S. Sharma, "Comparative review of evaluating and depleting energy hole problem in wireless sensor network," 2016 Online International Conference on Green Engineering and Technologies (IC-GET), Coimbatore, 2016, pp. 1-5.

[5] A. Sharma, R. Chaki and U. Bhattacharya, "Applications of wireless sensor network in Intelligent Traffic System: A review," 2011 3rd International Conference on Electronics Computer Technology, Kanyakumari, 2011, pp. 53-57. 
[6] S. A. Imam, A. Choudhary and V. K. Sachan, "Design issues for wireless sensor networks and smart humidity sensors for precision agriculture: A review," 2015 International Conference on Soft Computing Techniques and Implementations (ICSCTI), Faridabad, 2015, pp. 181-187.

[7] H. H. Kenchannavar, S. Beedakar and U. P. Kulkarni, "Optimization techniques to improve lifetime of wireless sensor networks: A review," 2015 International Conference on Energy Systems and Applications, Pune, 2015, pp. 446-450.

[8] T. Gui, C. Ma, F. Wang and D. E. Wilkins, "Survey on swarm intelligence based routing protocols for wireless sensor networks: An extensive study," 2016 IEEE International Conference on Industrial Technology (ICIT), Taipei, 2016, pp. 1944-1949.

[9] Bijaya Ketan Panigrahi, Yuhui Shi, Meng-Hiot Lim, Handbook of Swarm Intelligence: Concepts, Principles and Applications, Springer Science \& Business Media, 2011

[10] Jason Brownlee, Clever Algorithms: Nature-inspired Programming Recipes, Jason Brownlee, 2011

[11] S. Gupta, V. Sharma, N. Mohan, and P.S. Sandhu, "Color Reduction in RGB based on Bacteria Foraging Optimization", In International Conference on Computer and Communication Technologies (1CCCT '), 2012.

[12] S. Dasgupta, A. Biswas, S. Das, B. K. Panigrahi and A. Abraham, "A micro-bacterial foraging algorithm for highdimensional optimization," 2009 IEEE Congress on Evolutionary Computation, Trondheim, 2009, pp. 785-792.

[13] H. Chen, Y. Zhu, and K. Hu, "Adaptive bacterial foraging optimization", In Abstract and Applied Analysis, vol. 2011. Hindawi Publishing Corporation, 2011.

[14] Z-H. Wei, X. Zhao, K-W. Wang and Y. Xiong, "Bus dispatching interval optimization based on adaptive bacteria foraging algorithm", Mathematical Problems in Engineering, 2012

[15] A.N.K. Nasir, M.O.Tokhi, and N. Ghani, "Novel adaptive bacteria foraging algorithms for global optimization", Applied Computational Intelligence and Soft Computing, 2014

[16] R. Panda and M.K. Naik, "A crossover bacterial foraging optimization algorithm." Applied Computational Intelligence and Soft Computing,2012

[17] B.B. Mangaraj, M. R. Jena, and S.K. Mohanty, "Bacteria foraging algorithm in antenna design." Applied Computational Intelligence and Soft Computing, 2016

[18] L. Mao, Y. Mao, C. Zhou, C. Li, X. Wei, and H. Yang, "Particle swarm and bacterial foraging inspired hybrid artificial bee colony algorithm for numerical function optimization", Mathematical Problems in Engineering, 2016

[19] S. Monajemi, S. Sanei and S. H. Ong, "Advances in bacteria motility modelling via diffusion adaptation," 2014 22nd European Signal Processing Conference (EUSIPCO), Lisbon, 2014, pp. 2335-2339.

[20] S. Monajemi, S. Sanei, and S-H. Ong, "Advances in bacteria motility modelling via diffusion adaptation", In Signal Processing Conference (EUSIPCO), 2014 Proceedings of the 22nd European, pp. 2335-2339, 2014.

[21] M.S. Daas, S. Chikhi, and M. Batouche, "Bacterial foraging optimization with double role of reproduction and step adaptation", In Proceedings of the International Conference on Intelligent Information Processing, Security and Advanced Communication, pp. 71, 2015.

[22] A. K. Jain, S. C. Srivastava, S. N. Singh and L. Srivastava, "Bacteria Foraging Optimization Based Bidding Strategy Under Transmission Congestion," in IEEE Systems Journal, vol. 9, no. 1, pp. 141-151, March 2015.

[23] Y. Mishra, S. Mishra and F. Li, "Coordinated Tuning of DFIG-Based Wind Turbines and Batteries Using Bacteria Foraging Technique for Maintaining Constant Grid Power Output," in IEEE Systems Journal, vol. 6, no. 1, pp. 16-26, March 201

[24] M. A. Muñoz, S. K. Halgamuge, W. Alfonso and E. F. Caicedo, "Simplifying the Bacteria Foraging Optimization Algorithm," IEEE Congress on Evolutionary Computation, Barcelona, 2010, pp. 1-7

[25] N. A. Okaeme and P. Zanchetta, "Hybrid Bacterial Foraging Optimization Strategy for Automated Experimental Control Design in Electrical Drives," in IEEE Transactions on Industrial Informatics, vol. 9, no. 2, pp. 668-678, May 2013.

[26] C. Samanta, S. P. Panigrahi and B. K. Panigrahi, "Genetic-based bacteria foraging to optimise energy management of hybrid electric vehicles," in IET Electrical Systems in Transportation, vol. 4, no. 3, pp. 53-61, September 2014.

[27] A. A. A. Ari, A. Gueroui, N. Labraoui, B. O. Yenké, C. Titouna and I. Damakoa, "Adaptive scheme for collaborative mobile sensing in wireless sensor networks: Bacterial foraging optimization approach," 2016 IEEE 27th Annual International Symposium on Personal, Indoor, and Mobile Radio Communications (PIMRC), Valencia, 2016, pp. 1-6.

[28] P. Lalwani and S. Das, "Bacterial Foraging Optimization Algorithm for CH selection and routing in wireless sensor networks," 2016 3rd International Conference on Recent Advances in Information Technology (RAIT), Dhanbad, 2016, pp. 95-100.

[29] F. Li, Y. Zhang, J. Wu and H. Li, "Quantum bacterial foraging optimization algorithm," 2014 IEEE Congress on Evolutionary Computation (CEC), Beijing, 2014, pp. 1265-1272.

[30] B. Pitchaimanickam and S. Radhakrishnan, "Bacteria Foraging Algorithm based clustering in Wireless Sensor Networks," 2013 Fifth International Conference on Advanced Computing (ICoAC), Chennai, 2013, pp. 190-195.

[31] Q. Y. Zhao, M. Li, J. Luo, Y. Li and L. Dou, "A nanorobot swarming algorithm based on bacteria foraging optimization to eradicate cancer cells," 2014 IEEE International Conference on Robotics and Biomimetics (ROBIO 2014), Bali, 2014, pp. 2599-2604.

[32] Hemawathi.P and T G Basavaraju. Article: An Investigational Study of Energy Conservation Techniques in Hierarchical Routing Protocols in Wireless Sensor Network. International Journal of Computer Applications 101(7):14-19, September 2014 


\section{BIOGRAPHIES OF AUTHORS}

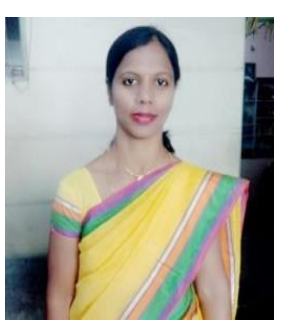

Hemavathi P obtained B.E from Manipal Institute of Technology, Manipal (University of MAHE), India. She completed M.Tech from Dr. Ambedkar Institute of Technology Bangalore, (VTU) India. Her areas of interest are Wireless Sensor Networks, Adhoc Networks. Currently she is pursuing Phd under Jain University, Bangalore.

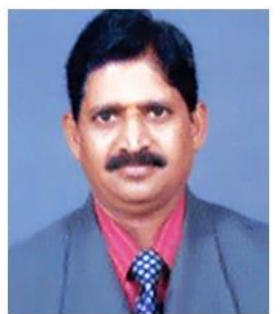

Dr. Nandakumar A N obtained his B.Sc in 1972, BE degree in 1976 both from university of Mysore, India and Ph.D from Berhanpur university in the year 2008 after getting M.Tech from University Of Roorkee (present IIT ROORKEE) in the year 1990. He is working as Professor, New horizon College of Engineering in the department of Computer science and engineering, Bangalore. His research is in the field of Image processing, pattern recognition, internet of things and others. He is a life member of ISTE. 